\title{
Kernos
}

Revue internationale et pluridisciplinaire de religion grecque antique

14 | 2001

Varia

\section{Les « héros » grecs dans tous leurs états}

Isabelle Ratinaud-Lachkar

\section{QpenEdition \\ Journals}

\section{Édition électronique}

URL : http://journals.openedition.org/kernos/784

DOI : $10.4000 /$ kernos.784

ISSN : 2034-7871

\section{Éditeur}

Centre international d'étude de la religion grecque antique

Édition imprimée

Date de publication : 1 janvier 2001

Pagination : 307-312

ISSN : 0776-3824

Référence électronique

Isabelle Ratinaud-Lachkar, "Les « héros » grecs dans tous leurs états », Kernos [En ligne], 14 | 2001, mis en ligne le 14 avril 2011, consulté le 24 septembre 2020. URL : http://journals.openedition.org/ kernos/784; DOI : https://doi.org/10.4000/kernos.784 


\title{
Revue des Livres
}

\section{Compte rendu critique}

\section{Les « héros » grecs dans tous leurs états ${ }^{1}$}

\author{
Isabelle Ratinaud-Lachkar (Université de Grenoble)
}

Consacré aux cultes héroïques, le cinquième séminaire suédois sur la religion grecque a réuni à Göteborg douze intervenants de différents horizons. Onze d'entre eux livrent ici le texte de leur communication, $R$. Hägg ne donnant que le résumé de la sienne (table des matières détaillée: Kernos, 13 [2000], p. 323). L'intérêt du volume ainsi élaboré est évident, dans la droite ligne des quatre précédents.

Les contributions frappent par leur diversité. Couvrant plus d'un millénaire de cultes héroïques, de l'âge du Fer (A. Mazarakis Ainian) à la période romaine (D.D. Hugues), elles s'appuient tant sur les vestiges archéologiques (C.M. Antonaccio, J.M. Hall) que sur les sources iconographiques (H.A. Shapiro, U. Kron) ou littéraires (C. Auffarth, U. Kron, B. McCauley, G. Ekroth). Les uns traitent des cultes héroïques à une époque bien précise (A. Mazarakis Ainian pour l'époque géométrique, D.D. Hugues pour les époques hellénistique et romaine), d'autres d'un aspect particulier des cultes héroïques à travers quelques exemples (U. Kron s'occupe de héros dits patriotiques, C.M. Antonaccio du lien entre les cultes héroïques et la colonisation, B. McCauley des politiques de transfert d'os de héros, J.M. Hall des rapports entretenus par les héros avec leur cité d'origine), d'autres encore d'un héros ou groupe de héros ( $\mathrm{C}$. Auffarth s'occupe des Danaïdes à Argos, H.A. Shapiro des Dioscures à Athènes). Certains enfin se penchent sur un site bien particulier (J.W. Riethmüller se rend à l'Asclépieion d'Athènes ${ }^{2}$, E.R. Gebhard \& M.W: Dickie au sanctuaire de l'Isthme) ou un auteur (G. Ekroth étudie le vocabulaire des sacrifices héroïques chez Pausanias). Deux index terminent l'ouvrage, l'un thématique, l'autre des sources littéraires.

1 A propos de Robin HäGG (éd.), Ancient Greek Hero Cult. Proceedings of the Fifth International Seminar on Ancient Greek Cult, organized by the Department of Classical Archaeology and Ancient History, Göteborg University, 21-23 April 1995, Stockholm, Áströms Förlag, 1999. 1 vol. $16,5 \times 24 \mathrm{~cm}, 207$ p. , ill. (Acta Instituti Atheniensis Regni Sueciae, Series in- $\left.8^{\circ}, 16\right)$. ISBN : 91-7916-037-9.

2 Je ne reviendrai pas sur la contribution de J.W. RIETHMÜLLER, objet d'un compterendu critique dans V. Pirenne-Delforge \& E. Suárez de la Torre (éds), Héros et héroïnes dans les mytbes et les cultes grecs. Actes du colloque du CIERGA, Valladolid, 26-29 mai 1999, Liège, 2000 (Kernos, suppl. 10), p. 329-332, sous la plume d'A. VerbancKPiÉrard, en annexe à sa communication consacrée aux cultes médicaux dans l'Attique classique, à laquelle je renvoie. 
Il n'est pas possible de reprendre ici dans le détail l'ensemble des onze contributions. Un certain nombre de remarques toutefois s'impose. Il est d'emblée regrettable que l'ouvrage ne s'ouvre pas sur une introduction thématique. La seule lecture de la table des matières en fait ressentir le besoin, besoin qui ne cesse ensuite de croître. Il ne s'agirait pas, certes, de résoudre les problèmes posés par l'apparente polysémie du mot " héros " à travers les siècles, mais du moins de les présenter, tous les auteurs n'en ayant visiblement pas la même acception. La lisibilité et l'impact de ce séminaire y auraient gagné. C'est en effet un des grands mérites de ce type d'ouvrage que de participer à la prise de conscience des difficultés, voire des contradictions nées de l'utilisation de ce terme, quand ce n'est pas de faire avancer la réflexion sur le phénomène héroïque. Ici, le héros est pris au sens le plus large qui soit.

A. Mazarakis Ainian et D.D. Hugues proposent, chacun pour une des bornes chronologiques de l'étude, un état des recherches assorti d'une riche bibliographie. A. Mazarakis Ainian présente pour l'âge du Fer un résumé des différentes théories des vingt-cinq dernières années, accompagné d'une présentation des principaux sites qui les étayent. L'auteur s'attarde sur les cultes en l'honneur des héros de l'épopée et de la mythologie puis sur les cultes funéraires et enfin sur les cultes en l'honneur des œcistes, selon une distinction très conventionnelle qui se justifie aujourd'hui de moins en moins faute de certitudes sur l'existence réelle de certaines de ces pratiques aux hautes époques. Il ne se cache pas (p. 9), et c'est tout à son honneur, qu'îl s'appuie largement pour ce faire sur un chapitre de son dernier ouvrage, From rulers' dwellings to temples: architecture, religion and society in Early Iron Age Greece (SIMA, 121), alors inédit. Notons, p. 14, l'insuffisance de l'argument : « in my opinion, the evidence of the character of the votives in (sic) not enough to deny the early date of the establishment of these hero shrines » pour refuser de remettre en cause l'attribution de lieux de culte d'époque géométrique à des héros de l'épopée. Car le contraire est tout aussi vrai. C'est donc là une véritable profession de foi !

Dans un tout autre esprit, la synthèse de D.D. Hugues, nécessairement rapide, n'en est pas moins efficace. Elle met en évidence l'évolution subie par le terme de "héros » à partir de l'époque hellénistique : le mot se vulgarise, les héroïsations publiques se multiplient cependant que des cultes plus anciens reviennent à l'honneur. Ces deux derniers phénomènes sont particulièrement sensibles durant le Haut Empire. Pausanias en serait-il le témoin?

C'est à ce sujet à une grande vigilance que nous appelle G. Ekroth. Sa magistrale et brillante démonstration est incontestablement un des points forts de cet ouvrage. En étudiant le vocabulaire utilisé par Pausanias pour décrire les sacrifices héroïques, la jeune chercheuse suédoise s'attaque à un problème central, le Périégète étant souvent dans ce domaine notre seule source. Par une démarche très rigoureuse, une recherche systématique, un texte structuré, étayé par des tableaux, G. Ekroth note d'abord combien Pausanias est un auteur atypique : lui

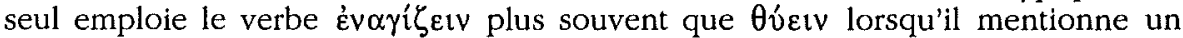
sacrifice héroïque. Qu'est-ce qui, sous la plume du Périégète, motive cette distinction? Il semble en fait que chacun des deux termes recouvre une réalité bien précise : Oúctv serait employé pour une pratique "normale », c'est-à-dire avec consommation de viande, $\dot{\varepsilon} v \alpha \gamma i \zeta \varepsilon \imath v$ pour une pratique exceptionnelle, la destruction complète de l'animal sacrifié. Force est alors de revenir sur les motivations de Pausanias : distinguant de fait plusieurs catégories de héros suivant qu'ils sont plus ou moins proches de la divinité (quand ils n'en sont pas simplement une, comme Héraclès), le Périégète note avant tout ce qui a lieu de l'être, donc l'exceptionnel, l'atypique. La « normalité » est alors quantitativement 
sous-estimée dans son œuvre. Précis, le Périégète est aussi homme de son temps : les pratiques décrites sont celles du $\mathrm{II}^{\mathrm{e}}$ siècle, époque marquée par une admiration profonde pour un passé remis à l'honneur (on retrouve là les principales conclusions de D.D. Hugues). Étude convaincante qui lève un coin du voile.

La contribution de E.R. Gebhard et M.W. Dickie consacrée au culte de Mélicerte au sanctuaire de l'Isthme en est considérablement fragilisée. Les deux auteurs partent du postulat que, malgré l'absence de tout vestige archéologique, il a dû exister là un culte à Mélicerte avant que ne soit aménagé le sanctuaire de Palaimon-Mélicerte, dont le premier état est daté du $\mathrm{I}^{\mathrm{er}}$ siècle de notre ère. Les auteurs appuient leur argumentation sur un unique fragment de Pindare constitué de trois vers. Non seulement ce fragment ne mentionne ni culte, ni sacrifice (il ne s'agit que de yépas, d'honneurs, institués par Sisyphe pour son enfant mort), mais encore les parallèles invoqués, littéraires et archéologiques, sont pour le moins peu convaincants. Dans la Ve Isthmique, il n'est question encore que de

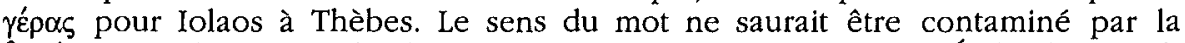
Ovoía, citée deux vers plus haut, pratiquée pour les Oenéides en Étolie (v. 30-35). Dans la $V^{e}$ Olympique, le verbe $\gamma \varepsilon p \alpha i p \varepsilon t v$ seul n'implique en rien un sacrifice

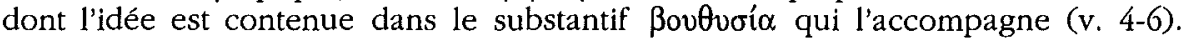
On ne saurait non plus s'appuyer sur un pseudo-culte à Opheltès à Némée : il n'en existe pas plus de trace que pour Mélicerte à l'Isthme, comme le font remarquer à juste titre les auteurs, et la tombe du héros au sanctuaire de Zeus n'est identifiée que par le seul Pausanias. Quant au culte de Pélops à Olympie tel qu'il serait décrit par Pindare dans sa $I^{\text {re }}$ Olympique ( $v$. 90-93), les dernières publications insistent sur le fait qu'à aucun moment Pindare n'établit de liens directs entre la tombe de Pélops et les sacrifices cependant que le $\beta \omega \mu$ ó s sur lequel sont pratiqués ces sacrifices est diversement interprété1. Les seuls vers de Pindare ne suffisent donc pas pour invoquer un culte rendu sur la tombe de Pélops à Olympie. Il ne s'agit pas, bien sûr, de nier le caractère funéraire des concours de l'Isthme institués en l'honneur de Mélicerte et leur renommée à travers le monde grec. Pindare et les scholiastes, notamment, s'en font l'écho et on peut effectivement établir là un parallèle avec Némée et Olympie. Mais il n'est besoin pour cela ni de la tombe d'un héros, ni d'un culte régulier en son honneur. L'existence d'un concours accompagné de sa légende n'implique pas la pratique d'un culte avec sacrifice. Par ailleurs, la présence d'une tombe ou d'un cénotaphe n'est en rien nécessaire au bon déroulement des concours. La quête d'un sanctuaire à Mélicerte antérieur à l'époque romaine me semble donc largement infondée. Rien ne laisse à penser qu'il faille contredire Pausanias et les données archéologiques qui concordent. Ne serait-il pas plus profitable de se demander pourquoi, au $\mathrm{I}^{\mathrm{er}}$ siècle, un Palaimonion a été ressenti comme nécessaire au sanctuaire de l'Isthme ${ }^{2}$ ?

Les six dernières communications portent sur les liens entre héros et politique, selon des modes très différents. S'interrogeant sur l'introduction du culte des Dioscures en Attique, H.A. Shapiro adopte une démarche riche d'enseignements. S'appuyant pour l'essentiel sur la représentation des Dioscures sur les vases attiques à partir de la deuxième moitié du $v^{e}$ siècle, l'auteur montre que,

1 Voir en dernier lieu G. Ekroth in Pirenne-Delforge \& SuÁrez de la Torre, o.c., p. 265-266, avec bibliographie.

2 Éléments de réponse in M. Piérart, "Panthéon et hellénisation dans la colonie romaine de Corinthe : la "redécouverte" du culte de Palaimon à l'Isthme", Kernos 11 (1998), p. 85-109. 
considérés d'abord comme des étrangers dont on voudrait faire des citoyens (scènes de théoxénies et de préparation des Dioscures à l'initiation d'Éleusis), Castor et Pollux sont peu à peu «atticisés », intégrés dans la mythologie athénienne (mis en scène avec Thésée comme protecteurs des pilotes, fonction essentielle pour l'Athènes du milieu $\mathrm{du}^{\mathrm{e}}$ siècle) jusqu'à la guerre du Péloponnèse. Les héros deviennent alors un enjeu entre Athènes, cité d'accueil, et Sparte, cité d'origine. L'étude s'arrête en 403. Une réserve seulement: les Athéniens, représentant les Dioscures invités à une théoxénie ou préparés pour l'initiation à Éleusis, veulent-ils en faire des citoyens athéniens, comme l'affirme l'auteur ( $p$. 101), ou insistent-ils plutôt sur leur hospitalité et par là sur l'origine grecque mais non-attique de leurs hôtes ? L'essentiel est que l'article mette clairement en évidence les possibilités d'évolution de la place d'un héros dans une cité, insistant sur le fait que, ni immuable ni acquis, le culte d'un héros, tout comme celui d'un dieu (pensons à Pan et à Asclépios à Athènes par exemple) peut être marqué par un contexte historique. Il y a dans le culte du héros une dimension politique qui l'a rendu particulièrement sensible aux aléas de l'Histoire durant toute l'Antiquité. Les articles suivants le confirment.

Pour son étude des héros dits patriotiques, U. Kron se penche sur les interventions de héros en vue de protéger, voire de sauver, une cité qui peut être la leur. L'article, sans plan clair ni conclusion, se présente comme un catalogue de cas examinés à la lueur de sources littéraires et iconographiques, fournissant parfois à son auteur loccasion de présenter différentes interprétations, sans toujours, au demeurant, prendre parti (ainsi p. 68). Sont alors décrites, entre autres,

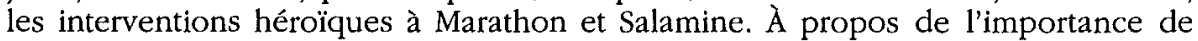
la présence des reliques, on ne saurait tirer aucune conclusion du seul cas du « héros » de la porte Ouest d'Érétrie (p. 73) que A. Mazarakis Ainian a discuté dans le même volume (p. 21). Dans un deuxième temps, l'auteur s'attarde sur les héros vénérés en tant que sauveur de leur cité, héros victorieux ou héros s'étant sacrifié : là est exposé l'exemple de Kodros avec une intéressante remarque sur l'intérêt nouveau que semble susciter le sanctuaire de ce héros en pleine guerre du Péloponnèse ( $\mathrm{p}$. 75-76). Il y a ainsi, dans cet article, à plusieurs reprises, matière à réflexion (à propos de Kodros par exemple, ou encore du récit par Plutarque de la mainmise athénienne sur Salamine, p. 69) mais les conclusions qui s'imposent ne viennent pas, faute peut-être d'une étude poussée du contexte historique dans chacun des cas : le projet était sans doute trop ambitieux pour un volume d'actes.

Dans son article très structuré et systématique consacré aux transferts d'os, B. McCauley développe deux exemples précis que l'on attendait : celui d'Oreste et celui de Thésée. Pour chacun d'entre eux, l'auteur s'interroge longuement : quel est le bénéfice escompté du transfert? ce bénéfice est-il lié à la personnalité du héros ? quel rôle joue ici le sanctuaire de Delphes ? pourquoi la présence des os des héros, non indispensables au culte, est-elle ressentie ici comme nécessaire? quelles croyances sous-tendent ces transferts? quelle en est alors la véritable signification? De nombreuses similitudes peuvent être relevées entre les deux événements, de même qu'avec les treize autres cas de transfert relevés par l'auteur. Les motivations politiques sont nettes la plupart du temps, souvent centrées sur des exigences territoriales. L'auteur rejoint ainsi certains aspects de l'article de U. Kron. La solide argumentation et le rigoureux raisonnement de B. McCauley n'ont qu'une faille : l'absence totale de considération pour la chronologie, alors que les faits examinés occupent plus de deux siècles. L'auteur termine sur un bref parallèle, pour le moins inattendu, avec le retour à Paris de la dépouille de Napoléon au $\mathrm{xIx}^{\mathrm{e}}$ siècle et celui aux Philippines de celle de Ferdinand Marcos en 1993, donnant ainsi un peu plus matière à réflexion. 
Partant de même du constat de la possible dimension politique des cultes héroïques, J.M. Hall s'étonne que des héros aussi "sensibles " qu'Hippolyte, les Sept ou Agamemnon puissent être vénérés dans plusieurs cités à la fois sans que les Grecs s'en soient apparemment émus. S'appuyant sur ces trois exemples, après avoir établi la liste de leurs lieux de culte, l'auteur met en évidence la disparité des situations. Le culte rendu à Hippolyte apparaît ainsi comme la matérialisation de relations d'amitié entre Athènes et Trézène qui l'honorent toutes deux. Celui des Sept, en tant que groupe, n'est pratiqué qu'à Argos qui l'aurait développé à partir du $\mathrm{v}^{\mathrm{e}}$ siècle dans le cadre d'une lutte d'influence contre une autre cité, Sparte ou Sicyone, sans pour autant en réclamer l'apanage : certains parmi les Sept, notamment Adraste et Amphiaraos, connaissent plusieurs autres lieux de culte. A propos du culte d'Agamemnon, J.M. Hall réaffirme son absence de Mycènes jusqu'au $\mathrm{r}^{\mathrm{e}}$ siècle, époque à laquelle est dévolu au héros le sanctuaire dit "Agamemnoneion ». Avant cette époque, on lui préfère Persée, véritable héros local. C'est à Sparte qu'il faudrait aller chercher les prémices d'un culte à Agamemnon. Voilà qui met à mal, de façon convaincante, nombre de théories, nourries d'exemples puisés dans la plaine argienne, sur les cultes héroïques d'époque géométrique comme partie intégrante de la naissance de la polis. On retrouve par ailleurs ici les dangers d'une lecture trop rapide de Pausanias qui avait fait pour nous le choix entre Mycènes et Sparte. $\AA$ la question « is it possible that Pausanias, like us, has been duped by the authority which is too often and too automatically ceded to the evidence of the Homeric epics?" (p. 55), je réponds sans hésitation par l'affirmative.

Le point de départ des réflexions de $C$. Auffarth peut sembler proche. L'auteur tente en effet de comprendre pourquoi la cité d'Argos a choisi les Danaïdes, marquées par tant de crimes, comme ancêtres. L'idée d'une telle recherche a de quoi surprendre. Mêlant sources littéraires, mythographiques et archéologiques sans considération ni pour le genre ni pour la chronologie (on ne peut sans quelques explications accepter de liens directs entre les récits homériques, certaines pratiques cultuelles de la plaine argienne et le témoignage de Pausanias, et encore ...), C. Auffarth conclut en six points dont cinq tombent sous le sens pour qui connaît un tant soit peu le mythe : on apprend ainsi que les Danaïdes constituent un groupe de vierges en marge de la communauté politique! Quant au sixième point, il n'engage que leur auteur : le mythe des Danaïdes aurait une origine phénicienne. Quand et comment a-t-il pu arriver à Argos ? La question n'est pas posée. L'auteur croit utile de préciser : "The Egyptian origin of the Danaides is not historical fact 》 (p. 48).

Le travail de C.M. Antonaccio aurait pu nous éclairer de façon décisive sur un aspect tout à fait primordial des cultes héroïques : leurs liens avec la colonisation. L'essentiel de l'article est en fait consacré à des considérations générales sur la colonisation, entrecoupées de longues citations et manquant parfois de précision. Les liens entre les colonies et les héros des nostoi sont envisagés par le biais des travaux d'Irad Malkin sur Cyrène. Les mêmes travaux permettent de mettre en valeur le seul exemple attesté de culte rendu à un œeciste, celui de Battos à Cyrène à partir de 630. L'auteur propose ensuite une troisième voie pour étudier les liens entre la colonisation grecque et les cultes hérö̈ques, l'examen des rapports que les Grecs entretenaient avec les tombes indigènes, mais reconnaît que les vestiges sont de lecture particulièrement délicate et ne permettent en fait aucune certitude. On en arrive alors à la conclusion que le culte de l'ociste relève du modèle littéraire et qu'il n'y a pas plus de culte héroïque dans les 
colonies que dans les métropoles ${ }^{1}$. Mais l'auteur ne s'y résout pas et voudrait faire des cultes héroïques la continuation des cultes des tombes et des cultes ancestraux. Les arguments pourtant manquent, les notions restent floues et les questions restent en suspens.

À l'issue de cette lecture, reconnaissons à nouveau l'intérêt du volume : toutes ces contributions apportent leur pierre à l'édifice, quitte à le fragiliser parfois. Dans l'état actuel des connaissances, il semble qu'un regard plus critique doive être jeté sur les sources, notamment littéraires, cependant que la vigilance quant à nos propres schémas doit être accrue. Notre attention est également attirée sur le vocabulaire et son évolution interne, sur le respect de la chronologie et la prise en compte de la durée. C'est à ce prix, semble-t-il, que progresseront les acquis sur le monde des héros. C'est le grand mérite de cet ouvrage que de nous y rendre plus sensibles, pour être plus clairyoyants.

Université Grenoble II

UFR de Sciences Humaines

BP 47

F - 38040 Grenoble Cedex 9

e-mail : Isabelle.Ratinaud@upmf-grenoble.fr

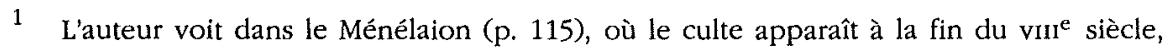
le premier exemple de sanctuaire hérö̈que dans le monde grec. Or le culte à Ménélas n'y semble pas pratiqué avant le vi ${ }^{\mathrm{e}}$ siècle. $C f$. I. Ratinaud-LachKar, "Héros homériques et sanctuaires d'époque géométrique", in Pirenne-Delforge \& SuÁrez de la Torre, o.c. (n. 2), p. 250-253. 